\section{Molecular Imaging: An Introduction}

H. Jadvar, H. Jacene, and M. Graham, Eds.

Cambridge, U.K.: Cambridge University Press, 2017, 82 pages, $\$ 49.99$

Molecular imaging is a relatively recent term. In 2007, the Society of Nuclear Medicine and Molecular Imaging formed a task force to develop standard definitions for this term and to serve as the foundation for all communications, advocacy, and education activities related to molecular imaging. The recommended definition for molecular imaging approved by the board of the Society of Nuclear Medicine and Molecular Imaging is the "visualization, characterization, and measurement of biologic processes at the molecular and cellular levels." The task force further elaborated that molecular imaging has relevance for patient care since it reveals the clinical biology of the disease process, personalizes patient care by characterizing specific disease processes, and is useful in drug discovery and development.

The aim of this small, handy book by 12 contributors is to provide a brief introduction to molecular imaging. It is not intended to be an exhaustive list of all available or potential imaging techniques or methods; rather, it includes only major modalities and applications, as comprehensive books covering detailed techniques and applications of molecular imaging are now available.

The book is organized into 15 chapters, starting with instrumentation and the physical principles of CT (chapter 1), MRI/MR spectroscopy (chapter 2), optical and ultrasound imaging (chapter 3 ), as well as nuclear medicine and PET (chapter 4). Chapter 5 deals with the quantitation of nuclear medicine imaging, and chapter 6 discusses myocardial and cerebral perfusion imaging. Chapter 7 covers metabolism of glucose, acetate, and choline. Chapter 8 deals with cellular proliferation, and chapter 9 addresses hypoxia. Chapter 10 discusses receptor imaging, and chapter 11 handles apoptosis. Chapter 12 covers angiogenesis, chapter 13 addresses reporter genes, chapter 14 discusses stem cell tacking, and the final chapter-chapter 15-deals with an amyloid imaging. The text is concise and well written, with clearly stated information. Each chapter begins with an introduction and concludes with a summary after a brief discussion. Some chapters provide current references for further reading. There are only 9 tables and 38 figures, and they are informative, but several of the images are not of high quality. The index is helpful.

This new introduction to molecular imaging enables the reader to effectively recognize the major methods, modalities, and various applications, providing insight for improved patient care, precision medicine, and medical research. Therefore, this book is useful for medical students, physicians in training, and others who desire to quickly grasp the basic concepts of molecular imaging.

\author{
E. Edmund Kim \\ University of California at Irvine \\ 101 The City Dr. $S$. \\ Orange, CA 92868 \\ E-mail: edmundek@uci.edu
}

